Behavioral/Systems/Cognitive

\title{
Glycinergic Projection Neurons of the Cerebellum
}

\author{
Martha W. Bagnall, ${ }^{1,2}$ Brian Zingg, ${ }^{2}$ Alexandra Sakatos, ${ }^{2}$ Setareh H. Moghadam, ${ }^{2}$ Hanns Ulrich Zeilhofer, ${ }^{4,5}$ \\ and Sascha du Lac ${ }^{1,2,3}$ \\ ${ }^{1}$ Neurosciences Graduate Program, University of California, San Diego, La Jolla, California 92093, ${ }^{2}$ Salk Institute for Biological Studies and ${ }^{3}$ Howard Hughes \\ Medical Institute, La Jolla, California 92037, ${ }^{4}$ Institute of Pharmacology and Toxicology, University of Zurich, CH-8057 Zurich, Switzerland, and 5 Institute \\ of Pharmaceutical Sciences, Eidgenössische Technische Hochschule Zurich, CH-8093 Zurich, Switzerland
}

The cerebellum funnels its entire output through a small number of presumed glutamatergic premotor projection neurons in the deep cerebellar nuclei and GABAergic neurons that feed back to the inferior olive. Here we use transgenic mice selectively expressing green fluorescent protein in glycinergic neurons to demonstrate that many premotor output neurons in the medial cerebellar (fastigial) nuclei are in fact glycinergic, not glutamatergic as previously thought. These neurons exhibit similar firing properties as neighboring glutamatergic neurons and receive direct input from both Purkinje cells and excitatory fibers. Glycinergic fastigial neurons make functional projections to vestibular and reticular neurons in the ipsilateral brainstem, whereas their glutamatergic counterparts project contralaterally. Together, these data suggest that the cerebellum can influence motor outputs via two distinct and complementary pathways.

\section{Introduction}

Vertebrates rely on the cerebellum for precision timing, coordinated motion, and cognition (Kim et al., 1994; Spencer et al., 2003). Despite the diverse functions of the cerebellum, all Purkinje cell axons from cerebellar cortex converge on a comparatively small number of neurons in the deep cerebellar and vestibular nuclei. Two classes of projection neurons have been described in the deep cerebellar nuclei: GABAergic neurons that provide feedback signals to the inferior olive, and glutamatergic neurons that modulate premotor and cortical circuits via projections to the brainstem, midbrain, and thalamus. These large premotor neurons receive input from both mossy fibers and Purkinje cells and constitute the sole means by which the cerebellum influences behavior. In addition, the deep nuclei are thought to be a site of enduring cerebellar plasticity (Aizenman et al., 2000; Ohyama et al., 2006; Pugh and Raman, 2006; Thompson and Steinmetz, 2009). Thus, it is critically important to understand how cerebellar nucleus neurons affect their downstream targets.

Although the literature refers exclusively to glutamatergic premotor (i.e., non-olivary) projection neurons of the deep cerebellar nuclei (Ito, 1984; Kandel et al., 1991; Squire et al., 2008), histological reports indicate the presence of large deep nuclear neurons immunopositive for glycinergic markers (Chen and Hillman, 1993; Bäurle and Grüsser-Cornehls, 1997; Tanaka and Ezure, 2004; Chung et al., 2009). GABAergic neurons projecting to the inferior olive are small to medium sized, whereas local interneurons are thought to be small (Chan-Palay, 1977; de Zeeuw et al., 1989; Fredette and Mugnaini, 1991; Batini et al.,

\footnotetext{
Received April 28, 2009; revised July 8, 2009; accepted July 11, 2009.

This work was supported by the Howard Hughes Medical Institute and National Institute of Health Grant EY11027 (S.d.L.) and a National Science Foundation Graduate Research Fellowship (M.W.B.). We thank Richard Jacobs for electron microscopy.

Correspondence should be addressed to Dr. Sascha du Lac, The Salk Institute for Biological Studies, 10010 North Torrey Pines Road, La Jolla, CA 92037. E-mail: sascha@salk.edu.

DOI:10.1523/JNEUROSCI.2087-09.2009

Copyright $\odot 2009$ Society for Neuroscience 0270-6474/09/2910104-07\$15.00/0
}

1992; De Zeeuw and Berrebi, 1995; Schwarz and Schmitz, 1997; Verveer et al., 1997; Teune et al., 1998; Uusisaari et al., 2007), raising the possibility that a subset of large projection neurons use glycine rather than glutamate as a neurotransmitter. Here we use a transgenic mouse line in which green fluorescence protein (GFP) is expressed under control of the GlyT2 (neuronal glycine transporter) promoter (Zeilhofer et al., 2005) to show that large glycinergic neurons in the fastigial nucleus are physiologically comparable with large glutamatergic projection neurons, are inhibited by Purkinje cells, and project outside the cerebellum to brainstem target regions.

\section{Materials and Methods}

Materials. Chemicals were from Sigma unless otherwise specified. Rabbit anti-calbindin was diluted 1:200 (Millipore Bioscience Research Reagents); cyanine 3 (Cy3) goat anti-rabbit (Millipore Bioscience Research Reagents) and Alexa 594 goat anti-rabbit (Invitrogen) were diluted 1:100. Two transgenic mouse lines were used: the GlyT2-GFP line, in which glycinergic neurons are labeled with the fluorescent reporter GFP (Zeilhofer et al., 2005), and L7-tau-GFP, in which Purkinje cells are selectively labeled with GFP (Sekirnjak et al., 2003). All experiments were performed in accordance with the Salk Institute Animal Care and Use Committee rules.

Surgery. Animals were deeply anesthetized with isoflurane until breathing slowed to approximately one breath per second and the footpinch reflex vanished. They were then placed on a stereotaxic apparatus with a bite bar (Benchmark Angle Two; http://MyNeuroLab.com) and given continuous inhalation anesthesia as needed to maintain status. In most cases, a custom-made injector needle $(0.2 \mathrm{~mm}$ outer diameter, 0.1 $\mathrm{mm}$ inner diameter; Creative Instruments Development Company) was loaded with crystals of fluoro-labeled dextran, either Texas Red, Cy3, or Cascade Blue (10,000 molecular weight; Invitrogen), and the tip was sealed with melted bone wax. In other surgeries, dye was dissolved in water or DMSO and delivered with a pulled glass pipette. Stereotaxic coordinates were used to guide injections into the ventromedial and ventrolateral nuclei of the thalamus $(n=5)$ and the lateral vestibular nucleus $(n=2)$. For medullary injections $(n=4)$, the midline was visually identified after blunt dissection of the neck muscles, and fiber 
tracts were targeted unilaterally $\sim 0.3 \mathrm{~mm}$ lateral to the midline, with a needle depth of $1.25 \mathrm{~mm}$. After the needle was lowered, the interior plunger was repeatedly depressed $(\sim 100 \mu \mathrm{m})$ with calibrated air pressure ( $25 \mathrm{psi}, 25 \mathrm{~ms}$ ) to deliver the crystals into the tissue. After waiting 1-2 min for the dye to settle, the needle was withdrawn and the skin was sutured. Animals were treated after surgery with Buprenex ( $1.5 \mu \mathrm{g}$ in saline) to minimize discomfort. Four to $8 \mathrm{~d}$ after injection, mice were killed.

Tissue preparation. Adult animals [older than postnatal day 28 (P28); typically 2-5 months old] from both GlyT2-GFP and L7-GFP mouse lines were anesthetized with Nembutal and perfused transcardially with PBS, followed by $4 \%$ paraformaldehyde in PBS (PFA) for 5 min. After removal of the brain from the skull, the tissue was postfixed for 30-60 min in PFA and then sunk in $30 \%$ sucrose in PBS overnight at $4^{\circ} \mathrm{C}$. Twenty to $50 \mu \mathrm{m}$ coronal or sagittal sections were cut on a freezing microtome (Microm) and washed in PBS or, in some cases, mounted directly onto slides. For immunocytochemistry of free-floating sections, blocking buffer ( $2 \%$ normal goat serum, $1 \%$ bovine serum albumin, and $0.3 \%$ Triton $\mathrm{X}-100$ in PBS) was applied for $1 \mathrm{~h}$, followed by primary antibody in working buffer (10-fold dilution of blocking buffer) overnight at $4^{\circ} \mathrm{C}$. Sections were washed three times with working buffer and treated with fluoro-conjugated secondary antibody for $1 \mathrm{~h}$ at room temperature. After washes in PBS, sections were wet-mounted and coverslipped with $2.5 \%$ DABCO (1,4-diazabicyclo-[2.2.2]octane) or Vectashield Hardset (Vector Laboratories).

For electron microscopic analyses, the paraformaldehyde-fixed brain of a 2-month-old (P50) GlyT2-GFP mouse was rinsed in cold PBS, and the cerebellum was cut into $50 \mu \mathrm{m}$ slices on a vibratome. Slices were imaged on a fluorescence microscope to locate large GFP-expressing neurons in the fastigial nucleus. Slices were then postfixed in $2 \%$ glutaraldehyde in $0.1 \mathrm{~m}$ sodium cacodylate buffer, rinsed, postfixed in $1 \%$ osmium tetroxide and $1 \%$ potassium ferrocyanide, rinsed, en bloc stained in 1\% uranyl acetate, dehydrated with glycol methacrylate, and flat embedded in Epon. The slices were blocked and mounted onto Epon stubs. Ultrathin sections $(\sim 60 \mathrm{~nm})$ were cut on an ultramicrotome, collected onto Formvar-coated slot grids, and stained with $2 \%$ uranyl acetate and $0.2 \%$ lead citrate. The sections were examined in a JEOL 100 CXII transmission electron microscope equipped with a digital camera. All chemicals were acquired from Electron Microscopy Sciences.

Image acquisition and processing. Epifluorescent images were recorded using a Hamamatsu CCD camera attached to a Olympus BX60 or BX61 light microscope with a $4 \times$ [numerical aperture (NA) 0.13 ] or $10 \times(\mathrm{NA}$ 0.3 ) objective lens with SlideBook 4. Confocal images were acquired in $0.1-0.5 \mu \mathrm{m}$ steps on a Leica TCS SP2 AOBS microscope using laser lines of 488 and $561 \mathrm{~nm}$, with a $20 \times($ NA 0.5$)$ or $63 \times$ (NA 1.4) objective and, in some cases, $3 \times$ hardware zoom. In most cases, images were collected by sequential scanning to avoid possible fluorophore crosstalk. Leica software was used to average sequential $z$-planes in images (two to six $z$-planes representing $<3 \mu \mathrm{m}$ total). Images were transferred to Adobe Photoshop (Adobe Systems) for whole-image brightness/contrast adjustment and image overlay.

Coronal cerebellar sections from three GlyT2-GFP mice were used for sizing analysis. Two of these contained retrogradely labeled neurons from dye injections in either the vestibular nuclei or the caudal medulla. Confocal images were acquired in $1 \mu \mathrm{m}$ steps with a $40 \times$ objective. Neuronal area and maximum diameter were assessed using Neurolucida. Nonprojecting neurons were randomly selected from the fastigial, interpositus, and dentate nuclei; retrogradely labeled neurons were identified in the fastigial nuclei. Throughout this paper, for ease of reference to the homologous nuclei in primates, we use the terms "fastigial," "interpositus," and "dentate" to refer to the medial, interposed, and lateral cerebellar nuclei, respectively.

Electrophysiology and reverse transcription-PCR. Coronal cerebellar slices were cut from GlyT2-GFP mice aged P10-P14; dense myelination in the deep nuclei and lateral vestibular nuclei in older animals is prohibitive for routine patch-clamp recording. Recordings were made at $\sim 33^{\circ} \mathrm{C}$ using a combination of epifluorescence and infrared illumination with differential interference contrast to visualize neurons. Data were collected and analyzed with custom-written code in Igor Pro 5. Ringer's solution for slicing and recording consisted of the following (in $\mathrm{mm}$ ):
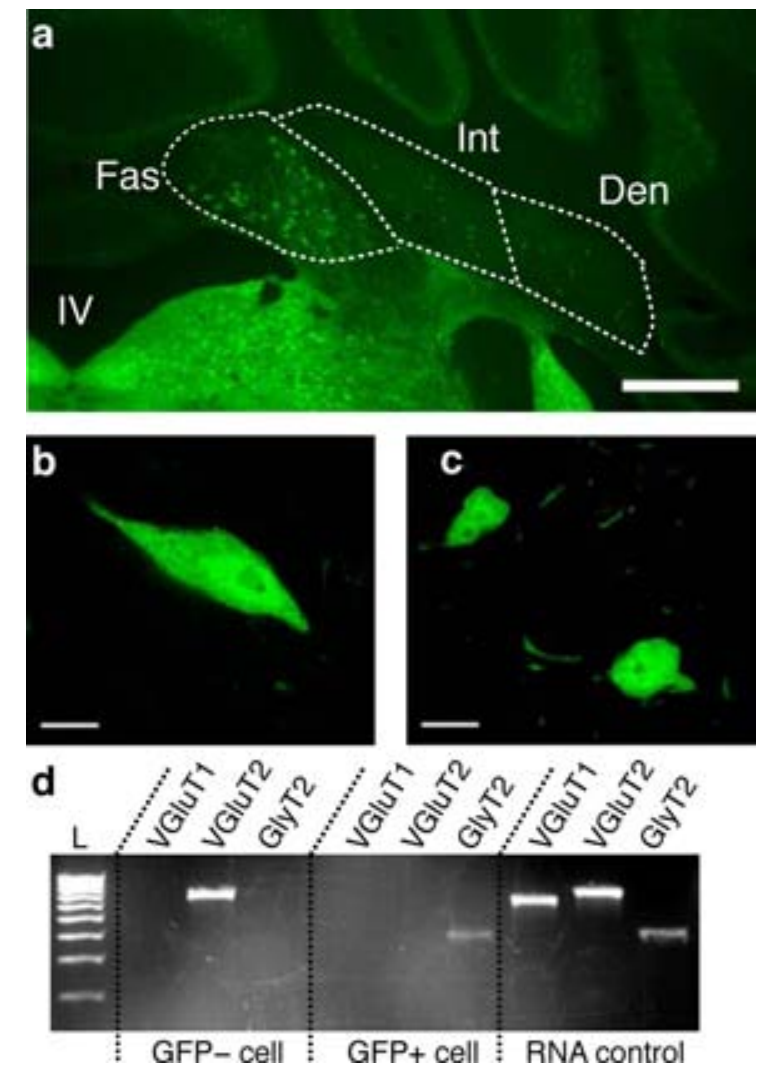

Figure 1. Identification of large glycinergic neurons in the fastigial nuclei. $\boldsymbol{a}$, Coronal section of cerebellum and brainstem in the GlyT2-GFP mouse. Large GFP ${ }^{+}$cells are evident in the fastigial (Fas) but not the interpositus (Int) or dentate (Den) deep cerebellar nuclei. Scattered cerebellar Golgi cells are also visible. IV, Fourth ventricle. Scale bar, $500 \mu \mathrm{m}$. $\boldsymbol{b}$, Confocal image of a large fastigial $\mathrm{GFP}^{+}$neuron; $\mathrm{c}$, small dentate $\mathrm{GFP}^{+}$presumed interneurons. Images represent several averaged z-planes totaling $<3 \mu \mathrm{m}$. Scale bars, $10 \mu \mathrm{m}$. $\boldsymbol{d}$, Single-cell RT-PCR reveals that large fastigial GFP ${ }^{-}$neurons express the glutamatergic marker VGluT2, whereas $\mathrm{GFP}^{+}$neurons express GlyT2. Right, Whole-brain RNA processed alongside experimental samples. Bottom ladder band is $200 \mathrm{bp}$, with increments of $100 \mathrm{bp}$. Performed as in the study by Bagnall et al. (2007).

$124 \mathrm{NaCl}, 26 \mathrm{NaHCO}_{3}, 5 \mathrm{KCl}, 1.3 \mathrm{MgCl}, 1 \mathrm{NaH}_{2} \mathrm{PO}_{4}$, and 11 dextrose. Pipette internal solution contained the following (in $\mathrm{mM}$ ): 140 K-gluconate, 10 HEPES, $8 \mathrm{NaCl}$, 0.1 EGTA, $2 \mathrm{MgATP}$, and $0.3 \mathrm{Na}_{2} \mathrm{GTP}$. In experiments examining synaptic projections, the following receptor antagonists were present as noted: 6,7-dinitroquinoxaline-2,3-dione (DNQX) $(10 \mu \mathrm{M})$ to block fast ionotropic glutamatergic transmission, D-aminophosphonovaleric acid $(25 \mu \mathrm{M})$ or $R$-3-(2carboxypiperazin-4yl)propyl-1-phosphonic acid (CPP) $(25 \mu \mathrm{M}$; Ascent Scientific) to block NMDA receptor-mediated transmission, strychnine $(1 \mu \mathrm{M})$ to block glycine receptors, and gabazine (SR95531 [2-(3-carboxypropyl)3-amino-6-(4-methoxyphenyl)pyridazinium bromide]; $10 \mu \mathrm{M}$ ) to block ionotropic GABAergic receptors. All synaptic data were acquired in the presence of these antagonists, with the appropriate blocker washed in at the conclusion of the experiment to verify transmitter identity. Latency is reported as the time to $10 \%$ of the peak IPSC. For excitatory inputs, neurons were clamped at approximately -75 to $-80 \mathrm{mV}$, and for inhibitory inputs, at -60 to $-50 \mathrm{mV}$ (junction potential corrected). Data are reported as means \pm SEM unless otherwise noted. Single-cell reverse transcription (RT)-PCR was performed as described previously (Bagnall et al., 2007) using primers to VGluT1, VGluT2, and GlyT2.

Statistics. Nonparametric statistics were used for all analyses of intrinsic physiological data. The Kruskal-Wallis test was used to determine multiple group comparison validity, followed by the Wilcoxon's unpaired test for between-group comparisons. Kruskal-Wallis values were as follows: maximum firing rate, 0.0029 ; input resistance, 0.00019 ; spike 
width, $<0.0001$. Synaptic responses to trains are presented as means \pm SEM. Cell size comparisons are presented as means $\pm \mathrm{SD}$.

\section{Results}

We identified glycinergic neurons in cerebellar tissue with a recently developed transgenic mouse line in which GFP is expressed under the promoter for the neuronal glycine transporter GlyT2 (Zeilhofer et al., 2005) (Fig. 1a). Many GlyT2-GFP-positive (GlyT2-GFP ${ }^{+}$) neurons in the rostral two-thirds of the fastigial nucleus, concentrated ventrally, were $\sim 20 \mu \mathrm{m}$ in diameter (Fig. 1b), comparable in size with the large projection neurons found in all three deep nuclei (Chan-Palay, 1977). Unilaterally, these neurons numbered $\sim 280$, or $\sim 15 \%$ of all large fastigial neurons (Heckroth, 1994) (GlyT2-GFP fastigial image series,supplementalFig. 1, availableatwww. jneurosci.org as supplemental material). In contrast, $\mathrm{GFP}^{+}$neurons in the interpositus and dentate deep nuclei were smaller, on average $\sim 12 \mu \mathrm{m}$ in diameter, indicating likely local interneurons (Fig. 1c) (Chan-Palay, 1977; Chen and Hillman, 1993) (see Fig. $4 d$, associated text).

To verify the accuracy of GFP expression, single neurons from the fastigial nuclei were subjected to RT-PCR for three neurotransmitter markers (Bagnall et al., 2007): the vesicular glutamate transporters VGluT1 and VGluT2, and GlyT2. Large GFP ${ }^{+}$neurons expressed GlyT2 but neither VGluT1 nor VGluT2 ( $n=7)$. In contrast, large GFP-negative $\left(\mathrm{GFP}^{-}\right)$neurons contained VGluT2 but not VGluT1 or GlyT2 $(n=4)$ (Fig. $1 d)$. Thus, although the majority of large fastigial neurons are, as expected, glutamatergic, the GlyT2-GFP transgenic line appropriately identifies a distinct set of large glycinergic neurons. To identify the role that these neurons play in the cerebellar circuit, we tested in turn the synaptic inputs and intrinsic processing properties of large Gly ${ }^{+}$ neurons.

Deep nuclear projection neurons receive two major types of input: dense GABAergic input from Purkinje cells in the cerebellar cortex, and glutamatergic input primarily from mossy fiber collaterals. Purkinje cell innervation was assessed at the light microscopic level using immunostaining for calbindin D-28k, which colocalizes with Purkinje cell terminals (Batini, 1990) as identified in the L7-tau-GFP transgenic mouse line, in which Purkinje cells selectively express GFP (Sekirnjak et al., 2003) (Fig. 2a). Calbindin immunostaining in GlyT2-GFP mice revealed that large fastigial glycinergic somata were surrounded by numerous Purkinje cell synaptic terminals, similar to the known innervation pattern in projection but not intrinsic neurons (Chan-Palay, 1977; Uusisaari and Knöpfel, 2008) (Fig. 2b). At the ultrastructural level, Purkinje cell terminals are recognizable by their large size, flattened vesicles, and multiple release sites (Chan-Palay, 1977; Telgkamp et al., 2004). Electron microscopy revealed the presence of many such axon terminals surrounding the soma of large glycinergic neurons (Fig. 2c). These boutons
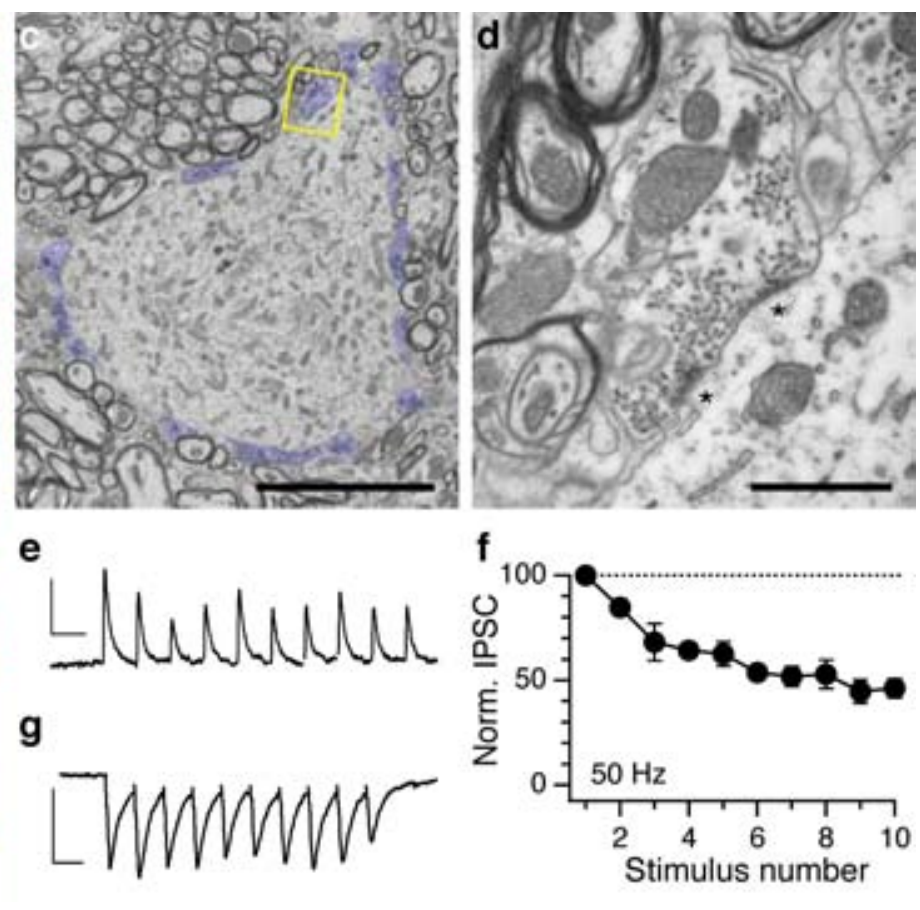

Figure 2. Purkinje cells inhibit large fastigial glycinergic neurons. $\boldsymbol{a}$, Calbindin immunostaining (top) identifies Purkinje cell terminals, visualized in the L7-GFP mouse (center) (Sekirnjak et al., 2003). Scale bar, $10 \mu \mathrm{m}$. $\boldsymbol{b}$, Calbindin immunostaining in the GlyT2-GFP line reveals that large fastigial glycinergic neurons are surrounded by Purkinje cell terminals. All ergicfar $f$ GABAergic currents exhibit sustained transmission at $50 \mathrm{~Hz}(n=4 \cdot \mathrm{mean}+\mathrm{SEM}) \mathrm{g}$ Glutamatergic synaptic currents (DNOX and (PP-sensitive) recorded in another large glycinergic fastigial neuron. Calibration: 100 pA, 20 ms.

contained multiple symmetric synapses and flattened vesicles typical of Purkinje cell GABAergic synapses (Fig. $2 d$ ).

Purkinje cell synapses exhibit several distinctive physiological characteristics, including sustained transmission at high frequencies (Telgkamp and Raman, 2002; Telgkamp et al., 2004). We made voltage-clamp recordings from large glycinergic neurons in slice preparation while stimulating the cerebellar vermis, the source of most Purkinje cell input to fastigial neurons. The resulting gabazine-sensitive synaptic current (Fig. 2e) was robust even at high frequencies of stimulation $(n=4)$ (Fig. $2 f)$, consistent with Purkinje cell synaptic characteristics (Telgkamp and Raman, 2002; Telgkamp et al., 2004). Thus, not all large Purkinje cell recipient neurons in the deep nuclei are glutamatergic, as had been thought previously (Chan-Palay, 1977; Batini et al., 1992; De Zeeuw and Berrebi, 1995; Teune et al., 1998).

Synaptic stimulation in the presence of blockers of ionotropic inhibitory transmission evoked fast inward currents that were abolished by application of glutamatergic receptor antagonists (Fig. $2 g$ ). These inputs presumably represent mossy fiber collaterals, which make excitatory synapses onto deep nuclear neurons; at the ultrastructural level, some synaptic terminals with typical excitatory characteristics (clear round vesicles, asymmetric synaptic densities) were also visible (data not shown). Therefore, large glycinergic neurons in the deep nuclei receive the same fundamental forms of circuit information as glutamatergic neurons.

In most systems, projection and local neurons exhibit different intrinsic physiological characteristics suited to their circuit function. Projection neurons and interneurons in the deep cere- 

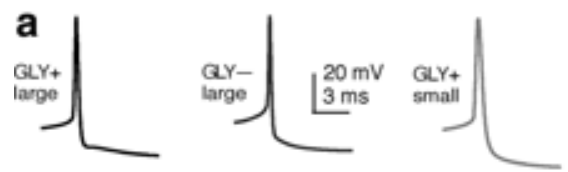

b

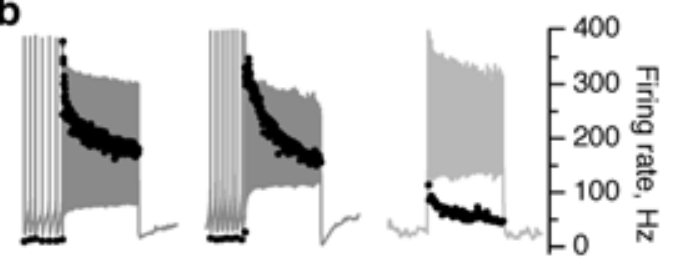

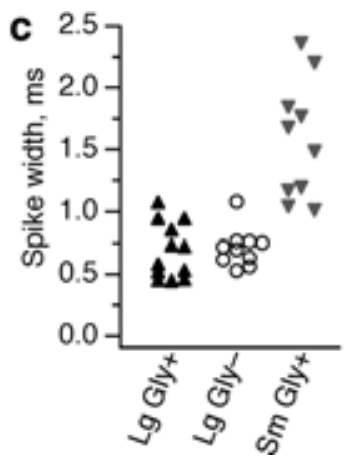

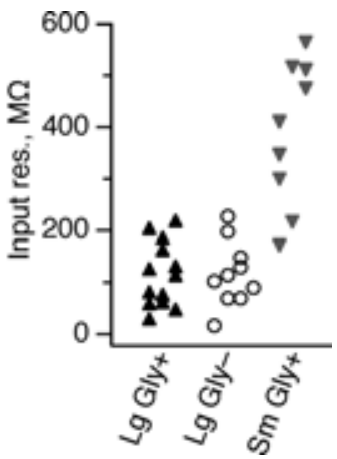

Figure 3. Physiological characteristics of large fastigial glycinergic neurons resemble those of large glutamatergic neurons, not small glycinergic neurons. $\boldsymbol{a}$, Action potential waveforms recorded from a typical large glycinergic neuron (left), large glutamatergic neuron (middle), and small glycinergic neuron (right). $\boldsymbol{b}$, Response to a $1 \mathrm{~s}$ step of depolarizing current at the maximum level to which the neuron could fire continuously across the whole step. Right axis, Instantaneous firing rate. c, Small glycinergic neurons are significantly different from both large glutamatergic and large glycinergic neurons in action potential width (left), maximum firing rate (middle), and input resistance (right) ( $p<0.05$, Wilcoxon's unpaired $t$ test, for all small vs large comparisons), whereas large glutamatergic and glycinergic neurons did not differ significantly ( $p>0.3$, all large glycinergic vs large glutamatergic comparisons).

bellar nuclei also differ in several essential features, including firing rate and action potential waveform (Jahnsen, 1986; Aizenman et al., 2003; Uusisaari et al., 2007). To determine whether large $\mathrm{Gly}^{+}$neurons more closely resemble their large excitatory or small inhibitory neighbors, we made recordings of all three types of neurons in cerebellar slices. Action potentials of both large glycinergic and glutamatergic neurons were less than half as broad as those of small glycinergic neurons (Fig. 3a,c). In response to a 1 s depolarizing current step, both $\mathrm{Gly}^{+}$and $\mathrm{Gly}^{-}$ large neurons were capable of firing action potentials at rates ranging from $\sim 100$ to 450 spikes/s, whereas maximum firing rates of $\mathrm{Gly}^{+}$small neurons saturated $<120$ spikes/s (Fig. 3b,c). In addition, subthreshold input resistance was significantly higher in small neurons than in either group of large neurons (Fig. 3c). Thus, the intrinsic properties of large glycinergic neurons are indistinguishable from those of large glutamatergic neurons but distinct from those of small glycinergic presumed interneurons. Notably, large $\mathrm{Gly}^{+}$neurons are also physiologically dissimilar from deep nuclear GABAergic neurons, in which action potentials are wider and firing saturates at $\sim 50$ spikes/s at room temperature (Uusisaari et al., 2007). Do large glycinergic neurons function as local cerebellar interneurons of unusual girth, or as premotor projection neurons, like the large glutamatergic neurons they resemble physiologically?

To evaluate whether $\mathrm{Gly}^{+}$fastigial neurons project to other brain areas, fluorescently conjugated dextrans were injected into target regions to label via retrograde transport any neurons projecting to or through the site of injection. After dye injection to the caudal ventral medulla, which labels axons projecting to the medullary reticular nuclei and spinal cord (Fig. 4a), confocal fluorescence microscopy revealed retrogradely labeled fastigial neurons in both halves of the fastigial nuclei, as expected (Matsushita and Hosoya, 1978; Asanuma et al., 1983). Interestingly, labeled fastigial neurons ipsilateral to the injection site were glycinergic (Fig. $4 b$ ), whereas those contralateral were glutamatergic (Fig. 4c) and segregated spatially in the dorsal half of the nucleus. Retrogradely labeled $\mathrm{Gly}^{+}$neurons from injections into the reticular formation or lateral vestibular nucleus were significantly larger than the population of $\mathrm{Gly}^{+}$neurons as a whole [long $\times$ short diameters: $\mathrm{Gly}^{+}$projection neurons, $189 \pm 67.5$ $\mu \mathrm{m}^{2}$ (mean $\pm \mathrm{SD}$ ); all $\mathrm{Gly}^{+}$neurons, $132 \pm 75.6 ; p<0.0001$, unpaired $t$ test], supporting the conclusion that large $\mathrm{Gly}^{+}$neurons make extracerebellar projections, whereas small Gly ${ }^{+}$neurons serve as local interneurons (Fig. $4 d$ ).
To localize specific targets of glycinergic outputs, we made unilateral dye injections directly into the fastigial nucleus. Glycinergic efferents traveled caudally in the ventral brainstem to the ipsilateral ventromedial medullary reticular formation (Asanuma et al., 1983; Homma et al., 1995), in which they issued terminal boutons that contacted both glycinergic and non-glycinergic neurons (Fig. 4e,f). Glycinergic efferents could also be followed to the ipsilateral lateral and descending vestibular nuclei (Fig. $4 g$ ), known targets of fastigial output (Homma et al., 1995). Dye injections into these target regions also resulted in retrograde labeling of ipsilateral glycinergic and contralateral glutamatergic fastigial neurons (data not shown). In contrast, thalamic tracer injection produced exclusively glutamatergic labeling of neurons that were concentrated in the caudal one-third of the contralateral fastigial nucleus (Fig. 4h), consistent with data from other species (Angaut and Bowsher, 1970; Sugimoto et al., 1981; Noda et al., 1990; Teune et al., 2000). Thus, it appears that fastigial glycinergic outputs are segregated to brainstem and caudal targets and do not extend rostrally.

To determine whether fastigial neurons identified in the GlyT2 transgenic mouse line actually release glycine onto their postsynaptic targets, we pursued physiological analysis of the fastigio-vestibular pathway. Thick coronal slices $(\sim 450 \mu \mathrm{m})$ from juvenile animals were used to maintain the fastigial nucleus, a section of cerebellar peduncle, and the lateral vestibular nucleus in one preparation (Fig. 5a). Whole-cell recordings were made from vestibular nucleus neurons while stimulating the fastigial nucleus in the presence of blockers of ionotropic glutamatergic and GABAergic synaptic transmission. The resulting outward synaptic current had a median latency of $1.5 \mathrm{~ms}$ and was fully blocked by the glycine receptor antagonist strychnine $(1 \mu \mathrm{M} ; n=$ 6) (Fig. 5b). Application of $50 \mathrm{~Hz}$ stimulus trains consistently drove synaptic facilitation, followed by sustained transmission $(n=5)$ (Fig. $5 c, d)$. Together, these data demonstrate a functional, ipsilateral glycinergic synaptic projection from the medial cerebellar nucleus to the brainstem.

\section{Discussion}

The cerebellum has been traditionally thought to influence the rest of the brain via two types of pathways emanating from the deep nuclei: a GABAergic feedback loop to the inferior olive and a glutamatergic projection to diverse brain regions. Our findings demonstrate the presence of a third, glycinergic pathway from the fastigial nucleus to the ipsilateral brainstem. It is matched by contralateral 
glutamatergic projections to the same vestibular and medullary reticular nuclei, providing a means for the cerebellum to coordinate midline musculature subserving balance and postural movements. Glycinergic and glutamatergic projection neurons are indistinguishable at the level of synaptic inputs and intrinsic physiology, indicating a common information processing scheme for excitatory and inhibitory premotor cerebellar nuclear neurons.

The fastigial nucleus has been divided into two functionally distinct parts that differ in neuronal response properties and projections (Ito, 1984; Thach et al., 1992). Rostral fastigial neurons are thought to mediate adaptive control of balance, posture, and autonomic function via their projections to brainstem vestibular and reticular nuclei. In contrast, caudal fastigial neurons are involved in cortical function and oculomotor control and project to the thalamus and to eye movementrelated portions of the brainstem and midbrain nuclei (Batton et al., 1977; Noda et al., 1990; Büttner et al., 1991). Their rostral location and postsynaptic targets in the vestibular nuclei and caudal brainstem implicate fastigial glycinergic projection neurons in balance and postural control.

Although the majority of deep cerebellar nucleus neurons project contralaterally, studies in a variety of species have demonstrated a prominent projection from the fastigial nucleus to the ipsilateral lateral and descending vestibular nuclei as well as a more modest projection to the ipsilateral medullary reticular formation (Ito, 1984). Our data confirm the presence of these pathways in mouse and identify the scattering of large glycinergic neurons observed in previous studies (Chen and Hillman, 1993; Bäurle and Grüsser-Cornehls, 1997; Tanaka and Ezure, 2004; Chung et al., 2009) as the major source of these ipsilateral projections. Inactivation of the rostral fastigial nucleus results in a tendency to fall toward the side of inactivation (Thach et al., 1992; Kurzan et al., 1993; Pélisson et al., 1998), as expected from a fastigial projection pattern of ipsilateral inhibition and contralateral excitation.

Why are glycinergic projection neurons found in the fastigial nucleus but not the interpositus and dentate? The parallel glycinergic and glutamatergic output channels of the rostral fastigial are well suited for directing behaviors that rely on cross-midline coordination, such as postural adjustments and gait. Interestingly, the vestibular control of horizontal eye movements is accomplished via ipsilateral glycinergic and contralateral glutamatergic outputs from the medial vestibular nucleus to the abducens ocular motor nuclei (Ito et al., 1977; Spencer et al., 1989).
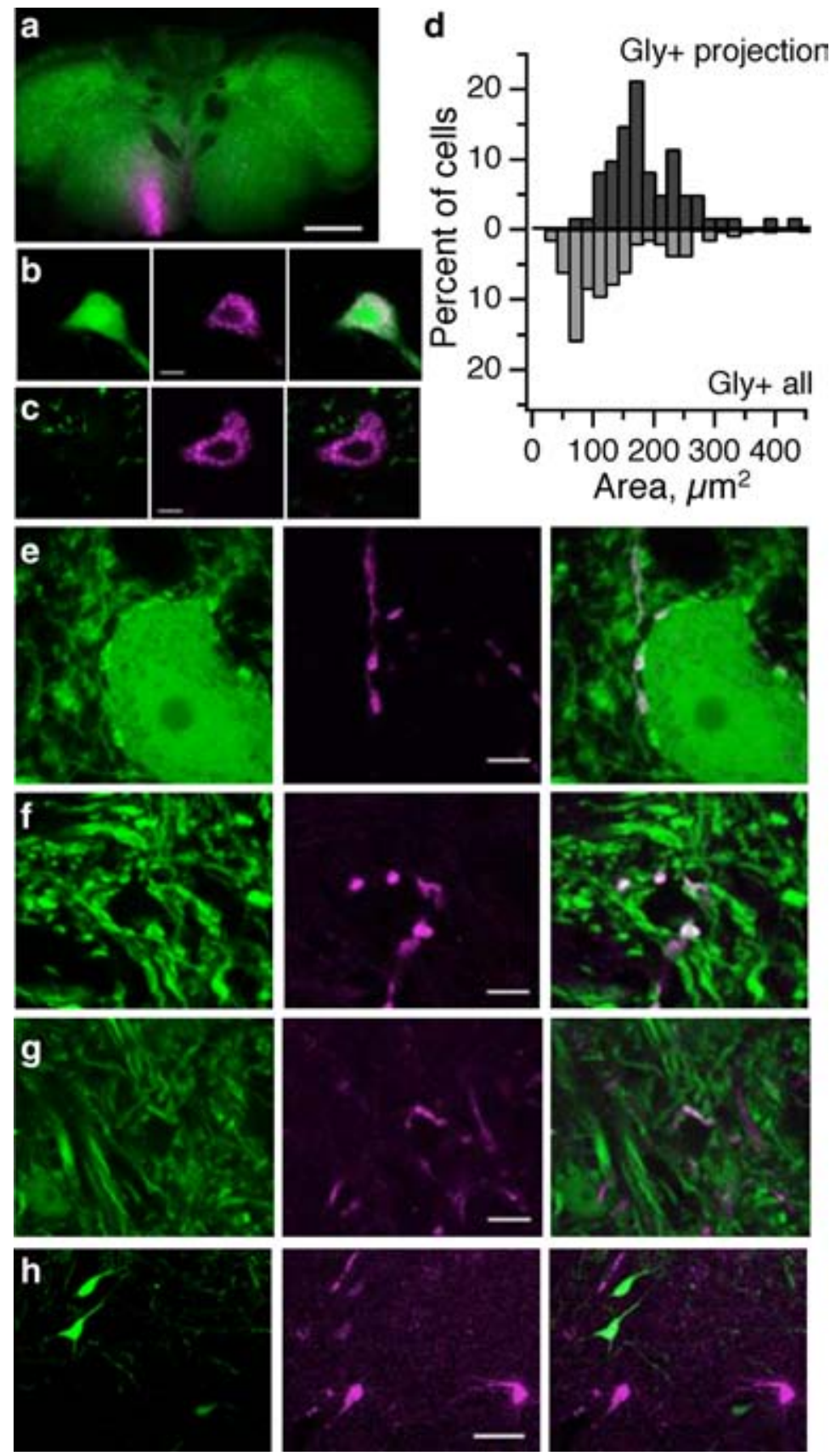

Figure 4. Glycinergic fastigial neurons project to the ipsilateral brainstem, whereas glutamatergic neurons project contralaterally. $\boldsymbol{a}$, Injection site in caudal medulla. Scale bar, $500 \mu \mathrm{m}$. $\boldsymbol{b}$, Ipsilateral to injection, retrogradely labeled fastigial neurons are glycinergic $\left(\mathrm{GFP}^{+}\right)$. In this and subsequent images: left, GFP expression; middle, retrograde labeling; right, merge. Scale bar, 10 $\mu \mathrm{m}$. $\boldsymbol{c}$, Contralateral to injection, retrogradely labeled fastigial neurons are glutamatergic. $\boldsymbol{d}$, Histogram distribution of somatic area of retrogradely labeled Gly ${ }^{+}$cells (top; $n=61$ neurons from several animals) versus all Gly ${ }^{+}$deep nuclear neurons (bottom; $n=132$ neurons). $\boldsymbol{e}, \boldsymbol{f}$, Anterogradely labeled glycinergic fastigial axons synapse onto glycinergic $(\boldsymbol{e})$ and non-glycinergic $(\boldsymbol{f})$ neurons in the ventromedial medullary reticular formation. $g$, Axonal swellings from glycinergic fastigial projections are also seen in the vestibular nuclei (here, the spinal vestibular nucleus). $\boldsymbol{h}$, No glycinergic retrogradely labeled neurons were seen after tracer injections to the thalamus. Scale bar, $50 \mu \mathrm{m}$.

We hypothesize that, during the evolutionary development of the fastigial nucleus, some of the horizontal vestibular bauplan was retained for coordination of midline musculature. In contrast, the later-evolving interpositus and dentate nuclei, which are critical for control of axial musculature, rely exclusively on glutamatergic premotor projections.

Several lines of evidence now demonstrate that the functional circuitry of the cerebellum, despite its crystalline architecture, is 
a

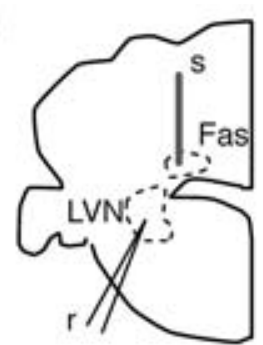

b Gabazine, DNQX,CPP

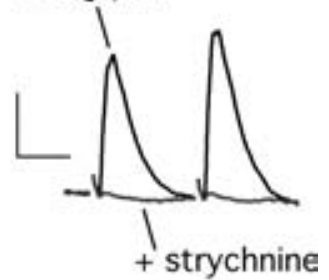

C

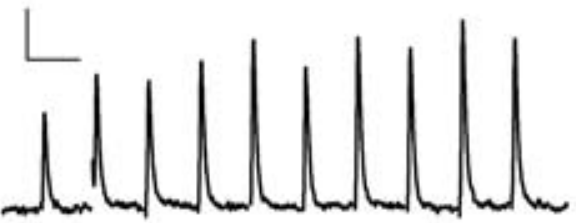

d

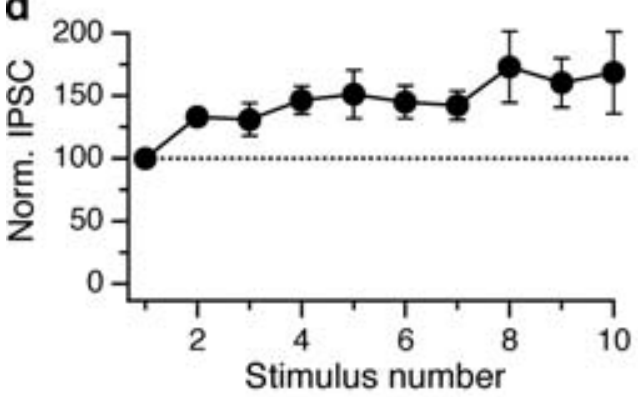

Figure 5. Functional inhibitory projection from the fastigial nucleus to the brainstem. $\boldsymbol{a}$, Diagram of the recording setup. Left half of a coronal section; top is dorsal, right is medial. A concentric bipolar stimulating electrode (s) was placed in the fastigial nucleus (Fas), and wholecell recordings (r) were made in the lateral vestibular nucleus (LVN). $\boldsymbol{b}$, The outward synaptic current recorded in a lateral vestibular nucleus neuron in the presence of ionotropic GABAergic and glutamatergic antagonists is abolished by application of strychnine $(1 \mu \mathrm{m})$. Calibration: 100 pA, 10 ms. Stimulus artifacts have been blanked. c, Response to a stimulus train at $50 \mathrm{~Hz}$ in another lateral vestibular nucleus neuron. The subtracted strychnine-sensitive component is shown. Calibration: $100 \mathrm{pA}, 20 \mathrm{~ms}$. $\boldsymbol{d}$, Fastigial glycinergic currents facilitated during a train of 10 stimuli delivered at $50 \mathrm{~Hz}(n=5$; mean \pm SEM).

not homogeneous. Parasagittal zones defined by the enzymatic marker zebrin II (aldolase C) (Leclerc et al., 1992) differ in expression of proteins and their subnuclear target localization (Sugihara and Shinoda, 2007), synaptic transmission parameters, and plasticity rules (Wadiche and Jahr, 2005). One type of excitatory interneuron, the unipolar brush cell, resides exclusively in the vestibulo-cerebellum (Dugué et al., 2005; Diana et al., 2007). Our findings indicate that premotor circuit connectivity differs across cerebellar nuclei, and that the midline fastigial nucleus contains both glutamatergic and glycinergic output channels.

The evidence presented here demonstrates that glycinergic and glutamatergic projection neurons in the fastigial nuclei share an essential role in the cerebellar circuit. They are indistinguishable in their intrinsic properties but physiologically distinct from small local glycinergic neurons, indicating that circuit role, rather than transmitter content per se, influences their processing properties, as has been shown for the medial vestibular nucleus (Bagnall et al., 2007).

Notably, all components of the cerebellar motor control circuit operate around high baseline firing rates. Both excitatory and inhibitory inputs to projection neurons are capable of sustaining high-frequency synaptic transmission (Fig. 2) (Telgkamp and Raman, 2002; Telgkamp et al., 2004). In vivo, rostral fastigial neurons fire spontaneously at 30-60 $\mathrm{Hz}$ (Büttner et al., 1991;
Miller et al., 2008), and both glycinergic and glutamatergic projection neurons can maintain high firing rates in vitro (Fig. 3). We provide the first evidence, to the best of our knowledge, that deep nuclear neurons evoke reliable synaptic currents that exhibit sustained transmission at physiological rates of activity (Fig. 5). Whether or not excitatory outputs from the deep nuclei exhibit similar properties remains to be determined (Gorodnov and Fanardjian, 1987; Jiang et al., 2002). Thus, rapid ongoing activity is a hallmark of cerebellar motor control and may be critical for fast, temporally precise modulation of movement.

Because the properties of glycinergic and glutamatergic neurons are so similar, it is likely that glycinergic and glutamatergic projection neurons are difficult to differentiate during in vivo experiments unless their precise projections are known; thus, much of the literature on fastigial activity likely includes data from both types of neurons. It is known that an individual Purkinje cell can make synaptic contacts onto both glutamatergic and GABAergic neurons (De Zeeuw and Berrebi, 1995). If the same is true for divergence onto both glutamatergic and glycinergic projection neurons, then a single Purkinje cell could influence coordinated bilateral movement by affecting both ipsilateral and contralateral premotor structures.

\section{References}

Aizenman CD, Huang EJ, Manis PB, Linden DJ (2000) Use-dependent changes in synaptic strength at the Purkinje cell to deep nuclear synapse. Prog Brain Res 124:257-273.

Aizenman CD, Huang EJ, Linden DJ (2003) Morphological correlates of intrinsic electrical excitability in neurons of the deep cerebellar nuclei. J Neurophysiol 89:1738-1747.

Angaut P, Bowsher D (1970) Ascending projections of the medial cerebellar (fastigial) nucleus: an experimental study in the cat. Brain Res 24:49-68.

Asanuma C, Thach WT, Jones EG (1983) Brainstem and spinal projections of the deep cerebellar nuclei in the monkey, with observations on the brainstem projections of the dorsal column nuclei. Brain Res 286:299-322.

Bagnall MW, Stevens RJ, du Lac S (2007) Transgenic mouse lines subdivide medial vestibular nucleus neurons into discrete, neurochemically distinct populations. J Neurosci 27:2318-2330.

Batini C (1990) Cerebellar localization and colocalization of GABA and calcium binding protein-D28K. Arch Ital Biol 128:127-149.

Batini C, Compoint C, Buisseret-Delmas C, Daniel H, Guegan M (1992) Cerebellar nuclei and the nucleocortical projections in the rat: retrograde tracing coupled to GABA and glutamate immunohistochemistry. J Comp Neurol 315:74-84.

Batton RR 3rd, Jayaraman A, Ruggiero D, Carpenter MB (1977) Fastigial efferent projections in the monkey: an autoradiographic study. J Comp Neurol 174:281-305.

Bäurle J, Grüsser-Cornehls U (1997) Differential number of glycine- and GABA-immunopositive neurons and terminals in the deep cerebellar nuclei of normal and Purkinje cell degeneration mutant mice. J Comp Neurol 382:443-458.

Büttner U, Fuchs AF, Markert-Schwab G, Buckmaster P (1991) Fastigial nucleus activity in the alert monkey during slow eye and head movements. J Neurophysiol 65:1360-1371.

Chan-Palay V (1977) Cerebellar dentate nucleus: organization, cytology and transmitters. Berlin: Springer.

Chen S, Hillman DE (1993) Colocalization of neurotransmitters in the deep cerebellar nuclei. J Neurocytol 22:81-91.

Chung SH, Marzban H, Hawkes R (2009) Compartmentalization of the cerebellar nuclei of the mouse. Neuroscience 161:123-138.

De Zeeuw CI, Berrebi AS (1995) Postsynaptic targets of Purkinje cell terminals in the cerebellar and vestibular nuclei of the rat. Eur J Neurosci 7:2322-2333.

de Zeeuw CI, Holstege JC, Ruigrok TJ, Voogd J (1989) Ultrastructural study of the GABAergic, cerebellar, and mesodiencephalic innervation of the cat medial accessory olive: anterograde tracing combined with immunocytochemistry. J Comp Neurol 284:12-35.

Diana MA, Otsu Y, Maton G, Collin T, Chat M, Dieudonné S (2007) T-type 
and L-type $\mathrm{Ca}^{2+}$ conductances define and encode the bimodal firing pattern of vestibulocerebellar unipolar brush cells. J Neurosci 27:3823-3838.

Dugué GP, Dumoulin A, Triller A, Dieudonné S (2005) Target-dependent use of coreleased inhibitory transmitters at central synapses. J Neurosci 25:6490-6498.

Fredette BJ, Mugnaini E (1991) The GABAergic cerebello-olivary projection in the rat. Anat Embryol 184:225-243.

Gorodnov VL, Fanardjian VV (1987) Functional properties of the cerebellorubral synapses in the cat. Brain Res 410:340-342.

Heckroth JA (1994) Quantitative morphological analysis of the cerebellar nuclei in normal and lurcher mutant mice. I. Morphology and cell number. J Comp Neurol 343:173-182.

Homma Y, Nonaka S, Matsuyama K, Mori S (1995) Fastigiofugal projection to the brainstem nuclei in the cat: an anterograde PHA-L tracing study. Neurosci Res 23:89-102.

Ito M (1984) The cerebellum and neural control. New York: Raven.

Ito M, Nisimaru N, Yamamoto M (1977) Specific patterns of neuronal connexions involved in the control of the rabbit's vestibulo-ocular reflexes by the cerebellar flocculus. J Physiol 265:833-854.

Jahnsen H (1986) Electrophysiological characteristics of neurones in the guinea-pig deep cerebellar nuclei in vitro. J Physiol 372:129-147.

Jiang MC, Alheid GF, Nunzi MG, Houk JC (2002) Cerebellar input to magnocellular neurons in the red nucleus of the mouse: synaptic analysis in horizontal brain slices incorporating cerebello-rubral pathways. Neuroscience 110:105-121.

Kandel ER, Schwartz JH, Jessell TM (1991) Principles of neural science, Ed 3. Norwalk, CT: Appleton and Lange.

Kim SG, Uğurbil K, Strick PL (1994) Activation of a cerebellar output nucleus during cognitive processing. Science 265:949-951.

Kurzan R, Straube A, Büttner U (1993) The effect of muscimol microinjections into the fastigial nucleus on the optokinetic response and the vestibulo-ocular reflex in the alert monkey. Exp Brain Res 94:252-260.

Leclerc N, Schwarting GA, Herrup K, Hawkes R, Yamamoto M (1992) Compartmentation in mammalian cerebellum: zebrin II and P-path antibodies define three classes of sagittally organized bands of Purkinje cells. Proc Natl Acad Sci U S A 89:5006-5010.

Matsushita M, Hosoya Y (1978) The location of spinal projection neurons in the cerebellar nuclei (cerebellospinal tract neurons) of the cat. A study with the horseradish peroxidase technique. Brain Res 142:237-248.

Miller DM, Cotter LA, Gandhi NJ, Schor RH, Huff NO, Raj SG, Shulman JA, Yates BJ (2008) Responses of rostral fastigial nucleus neurons of conscious cats to rotations in vertical planes. Neuroscience 155:317-325.

Noda H, Sugita S, Ikeda Y (1990) Afferent and efferent connections of the oculomotor region of the fastigial nucleus in the macaque monkey. J Comp Neurol 302:330-348.

Ohyama T, Nores WL, Medina JF, Riusech FA, Mauk MD (2006) Learninginduced plasticity in deep cerebellar nucleus. J Neurosci 26:12656-12663.

Pélisson D, Goffart L, Guillaume A (1998) Contribution of the rostral fastigial nucleus to the control of orienting gaze shifts in the headunrestrained cat. J Neurophysiol 80:1180-1196.

Pugh JR, Raman IM (2006) Potentiation of mossy fiber EPSCs in the cerebellar nuclei by NMDA receptor activation followed by postinhibitory rebound current. Neuron 51:113-123.

Schwarz C, Schmitz Y (1997) Projection from the cerebellar lateral nucleus to precerebellar nuclei in the mossy fiber pathway is glutamatergic: a study combining anterograde tracing with immunogold labeling in the rat. J Comp Neurol 381:320-334.
Sekirnjak C, Vissel B, Bollinger J, Faulstich M, du Lac S (2003) Purkinje cell synapses target physiologically unique brainstem neurons. J Neurosci 23:6392-6398.

Spencer RF, Wenthold RJ, Baker R (1989) Evidence for glycine as an inhibitory neurotransmitter of vestibular, reticular, and prepositus hypoglossi neurons that project to the cat abducens nucleus. J Neurosci 9:2718-2736.

Spencer RM, Zelaznik HN, Diedrichsen J, Ivry RB (2003) Disrupted timing of discontinuous but not continuous movements by cerebellar lesions. Science 300:1437-1439.

Squire LR, Bloom FE, Spitzer NC, du Lac S, Ghosh A, Berg D (2008) Fundamental neuroscience. San Diego: Academic.

Sugihara I, Shinoda Y (2007) Molecular, topographic, and functional organization of the cerebellar nuclei: analysis by three-dimensional mapping of the olivonuclear projection and aldolase C labeling. J Neurosci 27:9696-9710.

Sugimoto T, Mizuno N, Itoh K (1981) An autoradiographic study on the terminal distribution of cerebellothalamic fibers in the cat. Brain Res 215:29-47.

Tanaka I, Ezure K (2004) Overall distribution of GLYT2 mRNA-containing versus GAD67 mRNA-containing neurons and colocalization of both mRNAs in midbrain, pons, and cerebellum in rats. Neurosci Res 49:165-178.

Telgkamp P, Raman IM (2002) Depression of inhibitory synaptic transmission between Purkinje cells and neurons of the cerebellar nuclei. J Neurosci 22:8447-8457.

Telgkamp P, Padgett DE, Ledoux VA, Woolley CS, Raman IM (2004) Maintenance of high-frequency transmission at purkinje to cerebellar nuclear synapses by spillover from boutons with multiple release sites. Neuron 41:113-126.

Teune TM, van der Burg J, de Zeeuw CI, Voogd J, Ruigrok TJ (1998) Single Purkinje cell can innervate multiple classes of projection neurons in the cerebellar nuclei of the rat: a light microscopic and ultrastructural tripletracer study in the rat. J Comp Neurol 392:164-178.

Teune TM, van der Burg J, van der Moer J, Voogd J, Ruigrok TJ (2000) Topography of cerebellar nuclear projections to the brain stem in the rat. Prog Brain Res 124:141-172.

Thach WT, Goodkin HP, Keating JG (1992) The cerebellum and the adaptive coordination of movement. Annu Rev Neurosci 15:403-442.

Thompson RF, Steinmetz JE (2009) The role of the cerebellum in classical conditioning of discrete behavioral responses. Neuroscience. Advance online publication. Retrieved July 1, 2009. doi:10.1016/j.neuroscience.2009.01.041.

Uusisaari M, Knöpfel T (2008) GABAergic synaptic communication in the GABAergic and non-GABAergic cells in the deep cerebellar nuclei. Neuroscience 156:537-549.

Uusisaari M, Obata K, Knöpfel T (2007) Morphological and electrophysiological properties of GABAergic and non-GABAergic cells in the deep cerebellar nuclei. J Neurophysiol 97:901-911.

Verveer C, Hawkins RK, Ruigrok TJ, De Zeeuw CI (1997) Ultrastructural study of the GABAergic and cerebellar input to the nucleus reticularis tegmenti pontis. Brain Res 766:289-296.

Wadiche JI, Jahr CE (2005) Patterned expression of Purkinje cell glutamate transporters controls synaptic plasticity. Nat Neurosci 8:1329-1334.

Zeilhofer HU, Studler B, Arabadzisz D, Schweizer C, Ahmadi S, Layh B, Bösl MR, Fritschy JM (2005) Glycinergic neurons expressing enhanced green fluorescent protein in bacterial artificial chromosome transgenic mice. J Comp Neurol 482:123-141. 